\title{
Matter-wave optics: Observing an ultracold atomic cloud expanding in free fall
}

\author{
Wieland Schöllkopf ${ }^{1}$ \\ ${ }^{1}$ Fritz-Haber-Institut der Max-Planck-Gesellschaft
}

December 15, 2021

\begin{abstract}
This Highlight showcases the Research Paper entitled Collective-Mode Enhanced Matter-Wave Optics (Phys. Rev. Lett. 127, 100401 (2021), DOI: 10.1103/Phys- RevLett.127.100401).

\section{Hosted file}

NatSciences_Highlight_final.pdf available at https://authorea.com/users/451253/articles/ 549467-matter-wave-optics-observing-an-ultracold-atomic-cloud-expanding-in-free-fall

Hosted file

NatSciences_Highlight_final.tex available at https://authorea.com/users/451253/articles/ 549467-matter-wave-optics-observing-an-ultracold-atomic-cloud-expanding-in-free-fall
\end{abstract}


